\title{
Nonlinear Variation of Parameters Formula for Impulsive Differential Equations with Initial Time Difference and Application
}

\author{
Peiguang Wang ${ }^{1}$ and Xiaowei Liu $^{2,3}$ \\ ${ }^{1}$ College of Electronic and Information Engineering, Hebei University, Baoding 071002, China \\ ${ }^{2}$ College of Mathematics and Computer Science, Hebei University, Baoding 071002, China \\ ${ }^{3}$ College of Science, Hebei University of Engineering, Handan 056038, China \\ Correspondence should be addressed to Peiguang Wang; pgwang@mail.hbu.edu.cn
}

Received 27 February 2014; Accepted 19 May 2014; Published 5 June 2014

Academic Editor: Samir Saker

Copyright (C) 2014 P. Wang and X. Liu. This is an open access article distributed under the Creative Commons Attribution License, which permits unrestricted use, distribution, and reproduction in any medium, provided the original work is properly cited.

This paper establishes variation of parameters formula for impulsive differential equations with initial time difference. As an application, one of the results is used to investigate stability properties of solutions.

\section{Introduction}

It is now well recognized that impulsive differential equations are suitable mathematical models for many processes and phenomena in biology, physics, technology, and so forth. That is why in recent years the mathematical theory of such systems has gained increasing significance. We notice that most of the studies about initial value problems of impulsive differential equations are investigated only for perturbation or change of dependent variable keeping the initial time unchanged. However, in dealing with real world phenomena, it is impossible not to make errors in the starting time. When we consider such a change of initial time for each solution, we need to deal with the problem of comparing between any two solutions which start at different times.

At present, the investigation of differential systems with initial time difference has attracted a lot of attention. There are two methods of comparing the differences of the two solutions. One is the differential inequalities technique and comparison principle; the other is variation of parameters. For the pioneering works in this area we can refer to the papers [1, 2]. Ever since then, many results for various differential and difference systems have been obtained. The results obtained by the former method can be seen in [3-10]; and those done by the latter can be found in [11-15]. However, up till now, to the best of our knowledge, there are few results for impulsive differential equations with initial time difference. To be specific, there are no results on variation of parameters formula for impulsive differential equations relative to initial time difference. The method of variation of parameters is an important and fruitful technique since it is a practical tool in the investigation of the properties of solutions. It has been applied to the study of the relations of unperturbed and perturbed systems with different initial conditions.

In this paper, we will develop variation of parameters formula for impulsive differential equations with initial time changed and investigate Lipschitz stability by using one of the results obtained. The remainder of this paper is organized in the following manner. Some preliminaries are presented in Section 2, and various types of nonlinear variation of parameters formulae are established in Section 3. Finally, as an application, one of the results is applied to impulsive differential equations and the stability properties are obtained.

\section{Preliminaries}

Let $R^{+}=[0,+\infty)$ and let $R^{n}$ denote the $n$-dimensional Euclidean space with appropriate norm $\|\cdot\|$. 
Consider the following unperturbed impulsive differential equations

$$
\begin{aligned}
& x^{\prime}=f(t, x), \quad t \neq t_{k}, \\
& x\left(t_{0}^{+}\right)=x_{0}, \\
& x\left(t_{k}^{+}\right)=x\left(t_{k}\right)+I_{t_{k}}\left(x\left(t_{k}\right)\right), \\
& x^{\prime}=f(t, x), \quad t \neq t_{k}, \\
& x\left(\tau_{0}^{+}\right)=y_{0}, \\
& x\left(t_{k}^{+}\right)=x\left(t_{k}\right)+I_{t_{k}}\left(x\left(t_{k}\right)\right), \quad \text { whenever } t_{k} \geq \tau_{0},
\end{aligned}
$$

together with the perturbed ones of (2)

$$
\begin{aligned}
& y^{\prime}=F(t, y), \quad t \neq t_{k}, \\
& y\left(\tau_{0}^{+}\right)=y_{0}, \\
& y\left(t_{k}^{+}\right)=y\left(t_{k}\right)+I_{t_{k}}\left(y\left(t_{k}\right)\right), \quad \text { whenever } t_{k} \geq \tau_{0},
\end{aligned}
$$

where

(1) $0 \leq t_{0}<t_{1}<t_{2}<\cdots<t_{k}<\cdots$, and $\lim _{k \rightarrow \infty} t_{k}=$ $\infty, k=1,2, \ldots$;

(2) $\tau_{0}>t_{0}, \eta=\tau_{0}-t_{0}$;

(3) $\bar{t}_{k}=t_{k}-\eta \geq 0$;

(4) $S_{1}=\left\{t_{k}\right\}, S_{2}=\left\{\bar{t}_{k}\right\}, S=S_{1} \cup S_{2}$;

(5) $t \in R^{+}, x \in \Omega \subset R^{n}, \Omega$ - open;

(6) $f, F: R^{+} \times \Omega \rightarrow R^{n}$;

(7) $I_{t_{k}}: \Omega \rightarrow R^{n}$;

(8) $f(t, 0)=0, I_{t_{k}}(0)=0$, for all $t_{k}$.

We are concerned in this paper with the variation of parameters formula for impulsive differential equations relative to initial time difference. Before we can proceed, we will introduce the following lemmas [12], which are necessary for completing our main results.

Lemma 1. Let the following conditions be fulfilled:

$\left(A_{1}\right)$ the function $f: R^{+} \times \Omega \rightarrow R^{n}$ is continuous in $\left(t_{k-1}, t_{k}\right] \times \Omega, k=1,2, \ldots$ and for every $k$ and $x_{0} \in R^{n}$, there exists a finite limit of $f(t, x)$ as $(t, x) \rightarrow\left(t_{k}, x_{0}\right)$, $t>t_{k}$;

$\left(A_{2}\right)$ the function $f$ is locally Lipschitzian in $x$ on $R^{+} \times \Omega$;

$\left(A_{3}\right)$ for $k=1,2, \ldots$ the mapping $\psi_{k}: \Omega \rightarrow \Omega, x \rightarrow z$, $z=\psi_{k}(x) \equiv x+I_{k}(x)$ is a homeomorphism;

$\left(A_{4}\right)$ the system (1) had a solution $\phi(t)$ defined in $[\alpha, \beta]$, $\left(\alpha, \beta \neq t_{k}, k=1,2, \ldots\right)$.

Then there exist a number $\epsilon>0$ and a set

$$
V=\left\{(t, x) \in R^{+} \times \Omega, \alpha \leq t \leq \beta,\left|x-\phi\left(t^{+}\right)\right|<\epsilon\right\},
$$

such that, (i) for every $\left(t_{0}, x_{0}\right) \in V$, there exists a unique solution $x\left(t, t_{0}, x_{0}\right)$ of the system (1) which is defined on $[\alpha, \beta]$;

(ii) the function $x\left(t, t_{0}, x_{0}\right)$ is continuous for

$$
t \in[\alpha, \beta], \quad\left(t_{0}, x_{0}\right) \in V, \quad t, t_{0} \notin S_{1} ;
$$

(iii) for $k=1,2, \ldots, x_{0} \in \Omega, t, t_{0}$ belonging to the interval of existence of solution $x\left(t, t_{0}, x_{0}\right)$ of $(1), t \notin S_{1}$,

$$
\lim _{\substack{\xi \rightarrow t_{0} \\ \rho \rightarrow x_{0}}} x(t, \xi, \rho)=x\left(t, t_{0}, x_{0}\right) .
$$

Lemma 2. Let the following conditions be fulfilled:

$\left(A_{5}\right)$ the function $f: R^{+} \times \Omega \rightarrow R^{n}$ is continuous in $\left(t_{k-1}, t_{k}\right] \times \Omega, k=1,2, \ldots$, and $f_{x}(t, x)$ is continuous in $\left(t_{k-1}, t_{k}\right) \times \Omega, k=1,2, \ldots$;

$\left(A_{6}\right)$ for every $x_{0} \in \Omega, k=1,2, \ldots$, there exist finite limits of functions $f$ and $f_{x}$ as $(t, x) \rightarrow\left(t_{k}, x_{0}\right), t>t_{k}$;

$\left(A_{7}\right)$ for $k=1,2, \ldots$ the mapping $\psi_{k}: \Omega \rightarrow \Omega, x \rightarrow$ $z, z=\psi_{k}(x) \equiv x+I_{k}(x)$ is a diffeomorphism and for $x \in \Omega$

$$
\operatorname{det}\left(I+\frac{\partial I_{k}}{\partial x}(x)\right) \neq 0, \quad k=1,2, \ldots
$$

Then,

(i) there exists $\delta>0$ such that the solution $x\left(t, t_{0}, x_{0}\right)$ of (1) has continuous derivatives $\partial x / \partial t, \partial x / \partial t_{0}, \partial x / \partial x_{0}$, in the domain

$$
\begin{gathered}
V: \alpha<t<\beta, \quad \alpha<t_{0}<\beta, \quad t, t_{0} \neq t_{k}, \quad k=1,2, \ldots \\
\\
\left|x_{0}-\phi\left(t_{0}^{+}\right)\right|<\delta
\end{gathered}
$$

(ii) the derivative $\Phi\left(t, t_{0}, x_{0}\right)=\left(\partial x / \partial x_{0}\right)\left(t, t_{0}, x_{0}\right)$ is a solution of the initial value problem

$$
\begin{aligned}
& u^{\prime}=f_{x}(t, \phi(t)) u, \quad t \neq t_{k}, \\
& \Delta u=\frac{\partial I_{k}}{\partial x}\left(\phi\left(t_{k}\right)\right) u, \quad t=t_{k} \\
& u\left(t_{0}^{+}\right)=I
\end{aligned}
$$

where $\phi(t)$ is the solution of (1) in $[\alpha, \beta] \alpha, \beta \neq t_{k}, k=$ $1,2, \ldots$;

(iii) the derivative $\partial x / \partial t_{0}$ satisfies the relation

$$
\begin{aligned}
\frac{\partial x}{\partial t_{0}}\left(t, t_{0}, x_{0}\right) & =-\frac{\partial x}{\partial x_{0}}\left(t, t_{0}, x_{0}\right) f\left(t_{0}, x_{0}\right) \\
& =-\Phi\left(t, t_{0}, x_{0}\right) f\left(t_{0}, x_{0}\right)
\end{aligned}
$$




\section{Nonlinear Variation of Parameters Formula}

We will present, in this section, the nonlinear variation of parameters formula for impulsive differential equations relative to initial time difference. It is very useful for investigating the stability properties of solutions.

Theorem 3. Let the system (1) satisfy the conditions of Lemma 2 and let $x\left(t, t_{0}, x_{0}\right)$ be a solution of (1). Then for any solution $y(t)=y\left(t, \tau_{0}, y_{0}\right)$ of the system (3). The following formula is valid:

$$
\begin{aligned}
y(t+ & \left.\eta, \tau_{0}, y_{0}\right) \\
= & x\left(t, t_{0}, x_{0}\right)+\int_{0}^{1} \Phi\left(t, t_{0}, \sigma(s)\right)\left(y_{0}-x_{0}\right) d s \\
& +\int_{t_{0}}^{t} \tilde{f}(s, \tilde{y}(s), \eta) d s \\
& +\sum_{t_{0}<\bar{t}_{k}<t} \int_{0}^{1} \Phi\left(t, \bar{t}_{k}, \tilde{y}\left(\bar{t}_{k}\right)+s I_{\bar{t}_{k}+\eta}\left(\tilde{y}\left(\bar{t}_{k}\right)\right)\right) d s \\
& \cdot I_{\bar{t}_{k}+\eta}\left(\tilde{y}\left(\bar{t}_{k}\right)\right) .
\end{aligned}
$$

Proof. Set $p(s)=x(t, s, \tilde{y}(s))$, where $\tilde{y}(s)=y\left(s+\eta, \tau_{0}, y_{0}\right)$, $t_{0}<s<t$. Then for $s \notin S$, we have

$$
\begin{aligned}
p^{\prime}(s)= & \frac{\partial x}{\partial s}(t, s, \tilde{y}(s)) \\
& +\frac{\partial x}{\partial \tilde{y}}(t, s, \tilde{y}(s)) F(s+\eta, \tilde{y}(s)) \equiv \tilde{f}(s, \tilde{y}(s), \eta) .
\end{aligned}
$$

When $s \in S$, we have two cases.

Case 1. Consider

$$
\begin{aligned}
\Delta p & \left.(s)\right|_{s=\bar{t}_{k}} \\
& =x\left(t, \bar{t}_{k}^{+}, \tilde{y}\left(\bar{t}_{k}^{+}\right)\right)-x\left(t, \bar{t}_{k}^{-}, \tilde{y}\left(\bar{t}_{k}^{-}\right)\right) \\
& =x\left(t, \bar{t}_{k}, \tilde{y}\left(\bar{t}_{k}\right)+I_{\bar{t}_{k}+\eta}\left(\tilde{y}\left(\bar{t}_{k}\right)\right)\right)-x\left(t, \bar{t}_{k}, \tilde{y}\left(\bar{t}_{k}\right)\right) \\
& =\int_{0}^{1} \Phi\left(t, \bar{t}_{k}, \tilde{y}\left(\bar{t}_{k}\right)+s I_{\bar{t}_{k}+\eta}\left(\tilde{y}\left(\bar{t}_{k}\right)\right)\right) d s \cdot I_{\bar{t}_{k}+\eta}\left(\tilde{y}\left(\bar{t}_{k}\right)\right) .
\end{aligned}
$$

Case 2. Consider

$$
\left.\Delta p(s)\right|_{s=t_{k} \in S_{1}-S_{2}}=x\left(t, t_{k}^{+}, \tilde{y}\left(t_{k}^{+}\right)\right)-x\left(t, t_{k}^{-}, \tilde{y}\left(t_{k}^{-}\right)\right)=0 .
$$

Integrating (12) from $t_{0}$ to $t$ and using (13) and (14), we have

$$
\begin{aligned}
\tilde{y}(t)= & x\left(t, t_{0}, y_{0}\right)+\int_{t_{0}}^{t} \tilde{f}(s, \tilde{y}(s), \eta) d s \\
& +\sum_{t_{0}<\bar{t}_{k}<t} \int_{0}^{1} \Phi\left(t, \bar{t}_{k}, \tilde{y}\left(\bar{t}_{k}\right)+s I_{\bar{t}_{k}+\eta}\left(\tilde{y}\left(\bar{t}_{k}\right)\right)\right) d s \\
& \cdot I_{\bar{t}_{k}+\eta}\left(\tilde{y}\left(\bar{t}_{k}\right)\right) .
\end{aligned}
$$

Now let $q(s)=x\left(t, t_{0}, \sigma(s)\right)$, where $\sigma(s)=y_{0} s+(1-s) x_{0}$, $0 \leq s \leq 1$. Then we have

$$
\frac{d q(s)}{d s}=\frac{\partial x}{\partial \sigma}\left(t, t_{0}, \sigma(s)\right)\left(y_{0}-x_{0}\right)
$$

Integrating (16) from 0 to 1 , we arrive at

$$
x\left(t, t_{0}, y_{0}\right)=x\left(t, t_{0}, x_{0}\right)+\int_{0}^{1} \frac{\partial x}{\partial \sigma}\left(t, t_{0}, \sigma(s)\right)\left(y_{0}-x_{0}\right) d s .
$$

Combining (15) and (17) yields

$$
\begin{aligned}
y\left(t+\eta, \tau_{0}, y_{0}\right) & \\
= & x\left(t, t_{0}, x_{0}\right)+\int_{0}^{1} \Phi\left(t, t_{0}, \sigma(s)\right)\left(y_{0}-x_{0}\right) d s \\
& +\int_{t_{0}}^{t} \tilde{f}(s, \tilde{y}(s), \eta) d s \\
& +\sum_{t_{0}<\bar{t}_{k}<t} \int_{0}^{1} \Phi\left(t, \bar{t}_{k}, \tilde{y}\left(\bar{t}_{k}\right)+s I_{\bar{t}_{k}+\eta}\left(\tilde{y}\left(\bar{t}_{k}\right)\right)\right) d s \\
& \cdot I_{\bar{t}_{k}+\eta}\left(\tilde{y}\left(\bar{t}_{k}\right)\right) .
\end{aligned}
$$

The proof is complete.

Corollary 4. Suppose that the assumptions of Theorem 3 hold except that $F(t+\eta, y)$ being replaced with $f(t, y)+R(t+\eta, y)$; then the following formula is valid:

$$
\begin{aligned}
y\left(t+\eta, \tau_{0}, y_{0}\right) & \\
= & x\left(t, t_{0}, x_{0}\right)+\int_{0}^{1} \Phi\left(t, t_{0}, \sigma(s)\right)\left(y_{0}-x_{0}\right) d s \\
& +\int_{t_{0}}^{t} \Phi(t, s, \tilde{y}(s)) R(s+\eta, \tilde{y}(s)) d s \\
& +\sum_{t_{0}<\bar{t}_{k}<t} \int_{0}^{1} \Phi\left(t, \bar{t}_{k}, \tilde{y}\left(\bar{t}_{k}\right)+s I_{\bar{t}_{k}+\eta}\left(\tilde{y}\left(\bar{t}_{k}\right)\right)\right) d s \\
& \cdot I_{\bar{t}_{k}+\eta}\left(\tilde{y}\left(\bar{t}_{k}\right)\right) .
\end{aligned}
$$

Theorem 5. Suppose that the assumptions of Theorem 3 hold; then the following formula is valid:

$$
\begin{gathered}
y\left(t+\eta, \tau_{0}, y_{0}\right)-x\left(t, t_{0}, x_{0}\right) \\
=x\left(t, t_{0}, \eta\right)+\int_{t_{0}}^{t} \widetilde{H}(s, w(s), \eta) d s \\
+\sum_{\substack{t_{0}<\bar{t}_{k}<t \\
\bar{t}_{k} \in S_{2}-S_{1}}} \int_{0}^{1} \Phi\left(t, \bar{t}_{k}, y\left(\bar{t}_{k}+\eta\right)-x\left(\bar{t}_{k}\right)\right. \\
\left.\quad+s I_{\bar{t}_{k}+\eta}\left(y\left(\bar{t}_{k}+\eta\right)\right)\right) d s \\
\cdot I_{\bar{t}_{k}+\eta}\left(y\left(\bar{t}_{k}+\eta\right)\right)
\end{gathered}
$$




$$
\begin{gathered}
-\sum_{\substack{t_{0}<t_{k}<t \\
t_{k} \in S_{1}-S_{2}}} \int_{0}^{1} \Phi\left(t, t_{k}, y\left(t_{k}+\eta\right)-x\left(t_{k}\right)\right. \\
\left.\quad-s I_{t_{k}}\left(x\left(t_{k}\right)\right)\right) d s \cdot I_{t_{k}}\left(x\left(t_{k}\right)\right) \\
+\sum_{\substack{t_{0}<t_{k}<t \\
t_{k} \in S_{1} \cap S_{2}}} \int_{0}^{1} \Phi\left(t, t_{k}, y\left(t_{k}+\eta\right)-x\left(t_{k}\right)+s I_{t_{k}+\eta}\left(y\left(t_{k}+\eta\right)\right)\right. \\
\left.\cdot-s I_{t_{k}}\left(x\left(t_{k}\right)\right)\right) d s \\
\cdot\left(I_{t_{k}+\eta}\left(y\left(t_{k}+\eta\right)\right)-I_{t_{k}}\left(x\left(t_{k}\right)\right)\right) .
\end{gathered}
$$

Proof. Set $p(s)=x(t, s, w(s))$, where $w(s)=y\left(s+\eta, \tau_{0}, y_{0}\right)-$ $x\left(s, t_{0}, x_{0}\right), t_{0}<s<t$. Then for $s \notin S$, we have

$$
\begin{aligned}
p^{\prime}(s)= & \frac{\partial x}{\partial s}(t, s, w(s)) \\
& +\frac{\partial x}{\partial w}(t, s, w(s)) H(s, w(s), \eta) \equiv \widetilde{H}(s, w(s), \eta),
\end{aligned}
$$

where $H(s, w(s), \eta)=F(s+\eta, w(s)+x(s))-f(s, x(s))$.

If $s \in S$, we have three cases.

Case 1. Consider

$$
\begin{aligned}
&\left.\Delta p(s)\right|_{s=\bar{t}_{k} \in S_{2}-S_{1}} x\left(t, \bar{t}_{k}^{+}, w\left(\bar{t}_{k}^{+}\right)\right)-x\left(t, \bar{t}_{k}^{-}, w\left(\bar{t}_{k}^{-}\right)\right) \\
&= x\left(t, \bar{t}_{k}, y\left(\bar{t}_{k}^{+}+\eta\right)-x\left(\bar{t}_{k}^{+}\right)\right)-x\left(t, \bar{t}_{k}^{-}, y\left(\bar{t}_{k}^{-}+\eta\right)\right. \\
&\left.-x\left(\bar{t}_{k}^{-}\right)\right) \\
&=x\left(t, \bar{t}_{k}, y\left(\bar{t}_{k}+\eta\right)+I_{\bar{t}_{k}+\eta}\left(y\left(\bar{t}_{k}+\eta\right)\right)-x\left(\bar{t}_{k}\right)\right) \\
& \quad-x\left(t, \bar{t}_{k}, y\left(\bar{t}_{k}+\eta\right)-x\left(\bar{t}_{k}\right)\right) \\
&=\int_{0}^{1} \Phi\left(t, \bar{t}_{k}, y\left(\bar{t}_{k}+\eta\right)-x\left(\bar{t}_{k}\right)+s I_{\bar{t}_{k}+\eta}\left(y\left(\bar{t}_{k}+\eta\right)\right)\right) d s \\
& \quad \cdot I_{\bar{t}_{k}+\eta}\left(y\left(\bar{t}_{k}+\eta\right)\right) .
\end{aligned}
$$

Case 2. Consider

$$
\begin{aligned}
\left.\Delta p(s)\right|_{s=t_{k} \in S_{1}-S_{2}} & x\left(t, t_{k}^{+}, w\left(t_{k}^{+}\right)\right)-x\left(t, t_{k}^{-}, w\left(t_{k}^{-}\right)\right) \\
= & x\left(t, t_{k}, y\left(t_{k}^{+}+\eta\right)-x\left(t_{k}^{+}\right)\right)-x\left(t, t_{k}, y\left(t_{k}^{-}+\eta\right)-x\left(t_{k}^{-}\right)\right) \\
= & x\left(t, t_{k}, y\left(t_{k}+\eta\right)-I_{t_{k}}\left(x\left(t_{k}\right)\right)-x\left(t_{k}\right)\right) \\
& -x\left(t, t_{k}, y\left(t_{k}+\eta\right)-x\left(t_{k}\right)\right) \\
= & -\int_{0}^{1} \Phi\left(t, t_{k}, y\left(t_{k}+\eta\right)-x\left(t_{k}\right)-s I_{t_{k}}\left(x\left(t_{k}\right)\right)\right) d s \\
& \cdot I_{t_{k}}\left(x\left(t_{k}\right)\right) .
\end{aligned}
$$

Case 3. Consider

$$
\begin{aligned}
& \left.\Delta p(s)\right|_{s=t_{k} \in S_{1} \cap S_{2}} \\
& =x\left(t, t_{k}^{+}, w\left(t_{k}^{+}\right)\right)-x\left(t, t_{k}^{-}, w\left(t_{k}^{-}\right)\right) \\
& =x\left(t, t_{k}, y\left(t_{k}^{+}+\eta\right)-x\left(t_{k}^{+}\right)\right)-x\left(t, t_{k}, y\left(t_{k}^{-}+\eta\right)\right. \\
& \left.-x\left(t_{k}^{-}\right)\right)
\end{aligned}
$$

Integrating (21) from $t_{0}$ to $t$ and using (22) and (24), we have

$$
\begin{aligned}
y(t+ & \left.\eta, \tau_{0}, y_{0}\right)-x\left(t, t_{0}, x_{0}\right) \\
= & x\left(t, t_{0}, \eta\right)+\int_{t_{0}}^{t} \widetilde{H}(s, w(s), \eta) d s \\
& +\sum_{t_{0}<\bar{t}_{k}<t} \int_{0}^{1} \Phi\left(t, \bar{t}_{k}, y\left(\bar{t}_{k}+\eta\right)-x\left(\bar{t}_{k}\right)\right. \\
& -\sum_{\substack{t_{0}<S_{2}-S_{1}<t \\
t_{k} \in S_{1}-S_{2}}} \int_{0}^{1} \Phi\left(t, t_{k}, y\left(t_{k}+\eta\right)-x\left(t_{k}\right)-s I_{t_{k}}\left(x\left(t_{k}\right)\right)\right) d s \\
& \cdot I_{t_{k}}\left(x\left(t_{k}\right)\right) \\
& \left.+\sum_{\substack{t_{0}<t_{k}<t \\
t_{k} \in S_{1} \cap S_{2}}} \int_{0}^{1} \Phi\left(t, t_{k}, y\left(t_{k}+\eta\right)\right)\right) d s \cdot I_{\bar{t}_{k}+\eta}\left(y\left(\bar{t}_{k}+\eta\right)\right) \\
& \cdot\left(I_{t_{k}+\eta}\left(y\left(t_{k}+\eta\right)\right)-I_{t_{k}}\left(x\left(t_{k}\right)\right)\right) . \\
& \left.+s I_{t_{k}+\eta}\left(y\left(t_{k}+\eta\right)\right)-s I_{t_{k}}\left(x\left(t_{k}\right)\right)\right) d s \\
&
\end{aligned}
$$

The proof is complete.

\section{Application}

In this section, we turn to the Lipschitz stability of system (3):

$$
\begin{aligned}
y^{\prime} & =F(t, y), \quad t \neq t_{k}, \\
y\left(\tau_{0}^{+}\right) & =y_{0}, \\
y\left(t_{k}^{+}\right) & =y\left(t_{k}\right)+I_{t_{k}}\left(y\left(t_{k}\right)\right), \quad \text { whenever } t_{k} \geq \tau_{0},
\end{aligned}
$$

where $F(t+\eta, y)=f(t, y)+R(t+\eta, y)$. 
Definition 6. The solution $y\left(t+\eta, \tau_{0}, y_{0}\right)$ of the system (3) is said to be initial time difference Lipschitz stable (ITDLS) with respect to the solution $x\left(t, t_{0}, x_{0}\right)$ for $t \geq t_{0}$, where $x\left(t, t_{0}, x_{0}\right)$ is any solution of the system (1), if and only if there exists an $M=M\left(\tau_{0}\right)$ such that

$$
\left\|y\left(t+\eta, \tau_{0}, y_{0}\right)-x\left(t, t_{0}, x_{0}\right)\right\| \leq M\left(\left\|y_{0}-x_{0}\right\|+\tau_{0}-t_{0}\right) .
$$

Theorem 7. Let the following conditions be fulfilled:

$\left(B_{1}\right)$ the assumptions of Corollary 4 hold;

$\left(B_{2}\right)$ the zero solution of (1) is Lipschitz stable;

$\left(B_{3}\right)\|\Phi(t, s, \widetilde{y}(s)) R(s+\eta, \widetilde{y}(s))\| \leq \gamma(s)\|\widetilde{y}(s)\|$ for $t_{0}<s \leq t ;$

$\left(B_{4}\right)\left\|\Phi\left(t, t_{0}, \sigma(s)\right)\right\| \leq M_{1}\left(\left\|y_{0}-x_{0}\right\|+\eta\right) /\left\|y_{0}-x_{0}\right\|$ and $M_{1}$ is a constant;

$\left(B_{5}\right)\left\|I_{\bar{t}_{k}+\eta}\left(\widetilde{y}\left(\bar{t}_{k}\right)\right)\right\| \leq \beta_{k}\left\|\widetilde{y}\left(\bar{t}_{k}\right)\right\|$ and $\beta_{k} \geq 0$ are constants;

$\left(B_{6}\right)\left\|\Phi\left(t, \bar{t}_{k}, \tilde{y}\left(\bar{t}_{k}\right)+s I_{\bar{t}_{k}+\eta}\left(\tilde{y}\left(\bar{t}_{k}\right)\right)\right)\right\| \leq \alpha_{k}$ and $\alpha_{k} \geq 0$ are constants;

$\left(B_{7}\right) \int_{t_{0}}^{\infty} \gamma(s) d s<\infty, \gamma(s) \in C\left[R^{+}, R^{+}\right]$and $\prod_{t_{0}<\bar{t}_{k}<t}(1+$ $\left.\alpha_{k} \beta_{k}\right)<\infty$.

Then the solution $y\left(t+\eta, \tau_{0}, y_{0}\right)$ of the system (3) is ITDLS with respect to the solution $x\left(t, t_{0}, x_{0}\right)$.

Proof. From Corollary 4, it follows that

$$
\begin{aligned}
\tilde{y}(t) & -x\left(t, t_{0}, x_{0}\right) \\
= & \int_{0}^{1} \Phi\left(t, t_{0}, \sigma(s)\right)\left(y_{0}-x_{0}\right) d s \\
& +\int_{t_{0}}^{t} \Phi(t, s, \tilde{y}(s)) R(s+\eta, \tilde{y}(s)) d s \\
& +\sum_{t_{0}<\bar{t}_{k}<t} \int_{0}^{1} \Phi\left(t, \bar{t}_{k}, \tilde{y}\left(\bar{t}_{k}\right)+s I_{\bar{t}_{k}+\eta}\left(\tilde{y}\left(\bar{t}_{k}\right)\right)\right) d s \\
& \cdot I_{\bar{t}_{k}+\eta}\left(\tilde{y}\left(\bar{t}_{k}\right)\right) .
\end{aligned}
$$

Taking the norm and using the triangle inequality on both sides, we have

$$
\begin{aligned}
& \left\|\tilde{y}(t)-x\left(t, t_{0}, x_{0}\right)\right\| \\
& \leq \int_{0}^{1}\left\|\Phi\left(t, t_{0}, \sigma(s)\right)\right\|\left\|\left(y_{0}-x_{0}\right)\right\| d s \\
& \quad+\int_{t_{0}}^{t}\|\Phi(t, s, \tilde{y}(s)) R(s+\eta, \tilde{y}(s))\| d s \\
& \quad+\sum_{t_{0}<\bar{t}_{k}<t} \int_{0}^{1}\left\|\Phi\left(t, \bar{t}_{k}, \tilde{y}\left(\bar{t}_{k}\right)+s I_{\bar{t}_{k}+\eta}\left(\tilde{y}\left(\bar{t}_{k}\right)\right)\right)\right\| d s \\
& \quad \cdot\left\|I_{\bar{t}_{k}+\eta}\left(\tilde{y}\left(\bar{t}_{k}\right)\right)\right\| .
\end{aligned}
$$

From conditions $\left(B_{2}\right)-\left(B_{5}\right)$, we obtain

$$
\begin{aligned}
\| \tilde{y}(t) & -x\left(t, t_{0}, x_{0}\right) \| \\
\leq & M_{1}\left(\left\|y_{0}-x_{0}\right\|+\eta\right)+\int_{t_{0}}^{t} \gamma(s)\|\tilde{y}(s)\| d s \\
& +\sum_{t_{0}<\bar{t}_{k}<t} \alpha_{k} \beta_{k}\left\|\tilde{y}\left(\bar{t}_{k}\right)\right\| .
\end{aligned}
$$

Setting $M^{*}(t)=\left\|\tilde{y}(t)-x\left(t, t_{0}, x_{0}\right)\right\|$, we have

$$
\begin{aligned}
M^{*}(t) \leq & M_{1}\left(\left\|y_{0}-x_{0}\right\|+\eta\right)+\int_{t_{0}}^{t} \gamma(s) M^{*}(s) d s \\
& +\int_{t_{0}}^{t} \gamma(s)\left\|x\left(s, t_{0}, x_{0}\right)\right\| d s \\
& +\sum_{t_{0}<\bar{t}_{k}<t} \alpha_{k} \beta_{k} M^{*}\left(\bar{t}_{k}\right)+\sum_{t_{0}<\bar{t}_{k}<t} \alpha_{k} \beta_{k}\left\|x\left(\bar{t}_{k}\right)\right\| .
\end{aligned}
$$

Since $\left\|x\left(t, t_{0}, x_{0}\right)\right\| \leq M_{2}\left\|x_{0}\right\|$, as long as $\left\|x_{0}\right\|<\varepsilon$, then we have

$$
\begin{gathered}
\left\|x\left(t, t_{0}, x_{0}\right)\right\| \leq M_{2} \varepsilon \\
M^{*}(t) \leq M_{1}\left(\left\|y_{0}-x_{0}\right\|+\eta\right)+\int_{t_{0}}^{t} \gamma(s) M^{*}(s) d s \\
+M_{2} \varepsilon \int_{t_{0}}^{t} \gamma(s) d s+\sum_{t_{0}<\bar{t}_{k}<t} \alpha_{k} \beta_{k} M^{*}\left(\bar{t}_{k}\right) \\
+M_{2} \varepsilon \sum_{t_{0}<\bar{t}_{k}<t} \alpha_{k} \beta_{k} .
\end{gathered}
$$

Applying Gronwall's inequality to (32), we get

$M^{*}(t)$

$$
\begin{aligned}
& \leq\left\{M_{1}\left(\left\|y_{0}-x_{0}\right\|+\eta\right)\right. \\
& \left.\quad+M_{2} \varepsilon \int_{t_{0}}^{t} \gamma(s) d s+M_{2} \varepsilon \sum_{t_{0}<\bar{t}_{k}<t} \alpha_{k} \beta_{k}\right\} \\
& \quad \prod_{t_{0}<\bar{t}_{k}<t}\left(1+\alpha_{k} \beta_{k}\right) \exp \left\{\int_{t_{0}}^{t} \gamma(s) d s\right\} .
\end{aligned}
$$

Setting $M_{3}=\left\{M_{1}+\left(M_{2} \varepsilon \int_{t_{0}}^{t} \gamma(s) d s /\left\|y_{0}-x_{0}\right\|+\eta\right)+\left(M_{2}\right.\right.$ $\left.\left.\varepsilon \sum_{t_{0}<\bar{t}_{k}<t} \alpha_{k} \beta_{k} /\left\|y_{0}-x_{0}\right\|+\eta\right)\right\} \prod_{t_{0}<\bar{t}_{k}<t}\left(1+\alpha_{k} \beta_{k}\right) \exp \left\{\int_{t_{0}}^{t} \gamma(s) d s\right\}$, we have

$$
M^{*}(t) \leq M_{3}\left(\left\|y_{0}-x_{0}\right\|+\eta\right) .
$$

From condition $\left(B_{7}\right)$, it follows that the solution $y\left(t+\eta, \tau_{0}, y_{0}\right)$ of the system (3) is ITDLS with respect to the solution $x\left(t, t_{0}, x_{0}\right)$.

The proof is complete. 


\section{Conflict of Interests}

The authors declare that they have no competing interests.

\section{Authors' Contribution}

All authors completed the paper together. All authors read and approved the final paper.

\section{Acknowledgments}

The authors would like to thank the reviewers for their valuable suggestions and comments. This paper is supported by the National Natural Science Foundation of China (11271106) and the Natural Science Foundation of Hebei Province of China (A2013201232).

\section{References}

[1] V. Lakshmikantham and A. S. Vatsala, "Differential inequalities with initial time difference and applications," Journal of Inequalities and Applications, vol. 3, no. 3, pp. 233-244, 1999.

[2] V. Lakshmikantham, S. Leela, and J. V. Devi, "Another approach to the theory of differential inequalities relative to changes in the initial times," Journal of Inequalities and Applications, vol. 4, no. 2, pp. 163-174, 1999.

[3] T. Jankowski, "Systems of differential inequalities with initial time difference," Ukrainian Mathematical Journal, vol. 56, no. 1, pp. 139-145, 2004.

[4] T. Jankowski, "Integro-differential inequalities with initial time difference and applications," Acta Mathematica Hungarica, vol. 100, no. 4, pp. 329-342, 2003.

[5] T. Jankowski, "Differential inequalities with initial time difference," Applicable Analysis, vol. 81, no. 3, pp. 627-635, 2002.

[6] F. A. McRae, "Perturbing Lyapunov functions and stability criteria for initial time difference," Applied Mathematics and Computation, vol. 117, no. 2-3, pp. 313-320, 2001.

[7] Y. Zhang and B. Zhang, "Impulsive differential equations with initial time difference and applications," Dynamics of Continuous, Discrete \& Impulsive Systems A. Mathematical Analysis, vol. 9, no. 3, pp. 439-447, 2002.

[8] C. Yakar and M. D. Shaw, "Practical stability in terms of two measures with initial time difference," Nonlinear Analysis: Theory, Methods and Applications, vol. 71, no. 12, pp. 781-785, 2009.

[9] C. Yakar, "Fractional differential equations in terms of comparison results and Lyapunov stability with initial time difference," Abstract and Applied Analysis, vol. 2010, Article ID 762857, 16 pages, 2010.

[10] J. Y. Bao, P. G. Wang, and C. X. Gao, "Stability criteria for differential equations with initial time difference," Acta Mathematicae Applicatae Sinica, vol. 35, no. 4, pp. 608-616, 2012.

[11] M. D. Shaw and C. Yakar, "Generalized variation of parameters with initial time difference and a comparison result in term Lyapunov-like functions," International Journal of Non-Linear Differential Equations-Theory Methods and Applications, vol. 5, pp. 86-108, 1999.

[12] C. Yakar and S. G. Deo, "Variation of parameters formulae with initial time difference for linear integrodifferential equations," Applicable Analysis, vol. 85, no. 4, pp. 333-343, 2006.
[13] M. D. Shaw and C. Yakar, "Stability criteria and slowly growing motions with initial time difference," Problems of Nonlinear Analysis in Engineering Systems, vol. 1, pp. 50-66, 2000.

[14] C. Yakar and M. D. Shaw, "Initial time difference stability in terms of two measures and a variational comparison result," Dynamics of Continuous, Discrete \& Impulsive Systems A. Mathematical Analysis, vol. 15, no. 3, pp. 417-425, 2008.

[15] V. Lakshmikantham, S. Leela, and J. V. Devi, "Stability criteria for solutions of differential equations relative to initial time difference," International Journal of Nonlinear Differential Equations, vol. 5, pp. 109-114, 1999. 


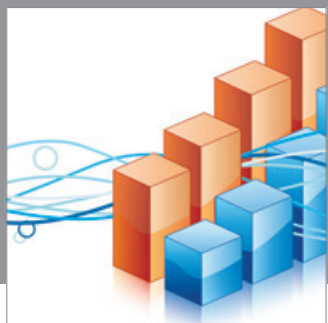

Advances in

Operations Research

mansans

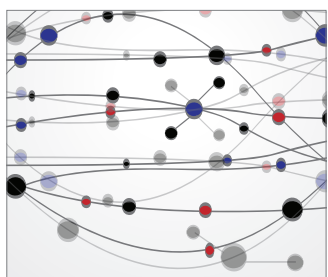

The Scientific World Journal
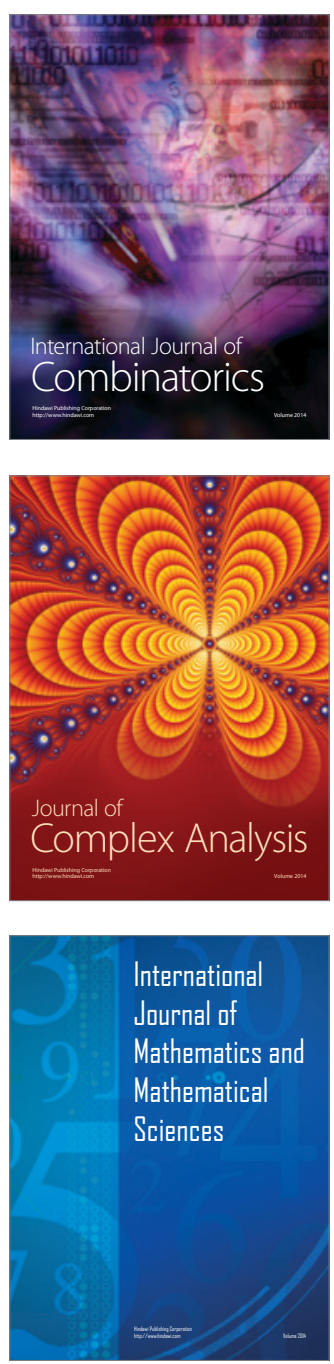
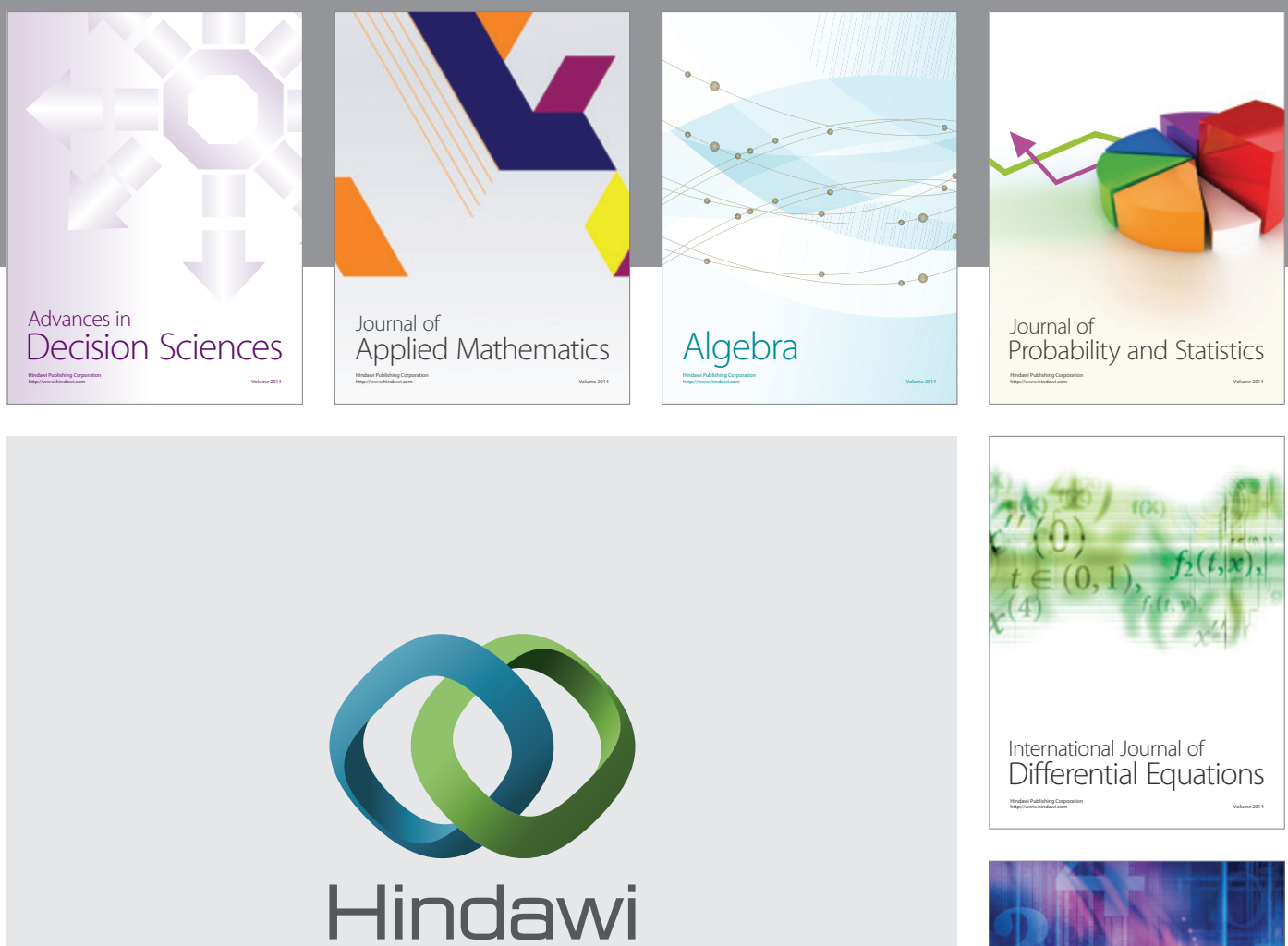

Submit your manuscripts at http://www.hindawi.com
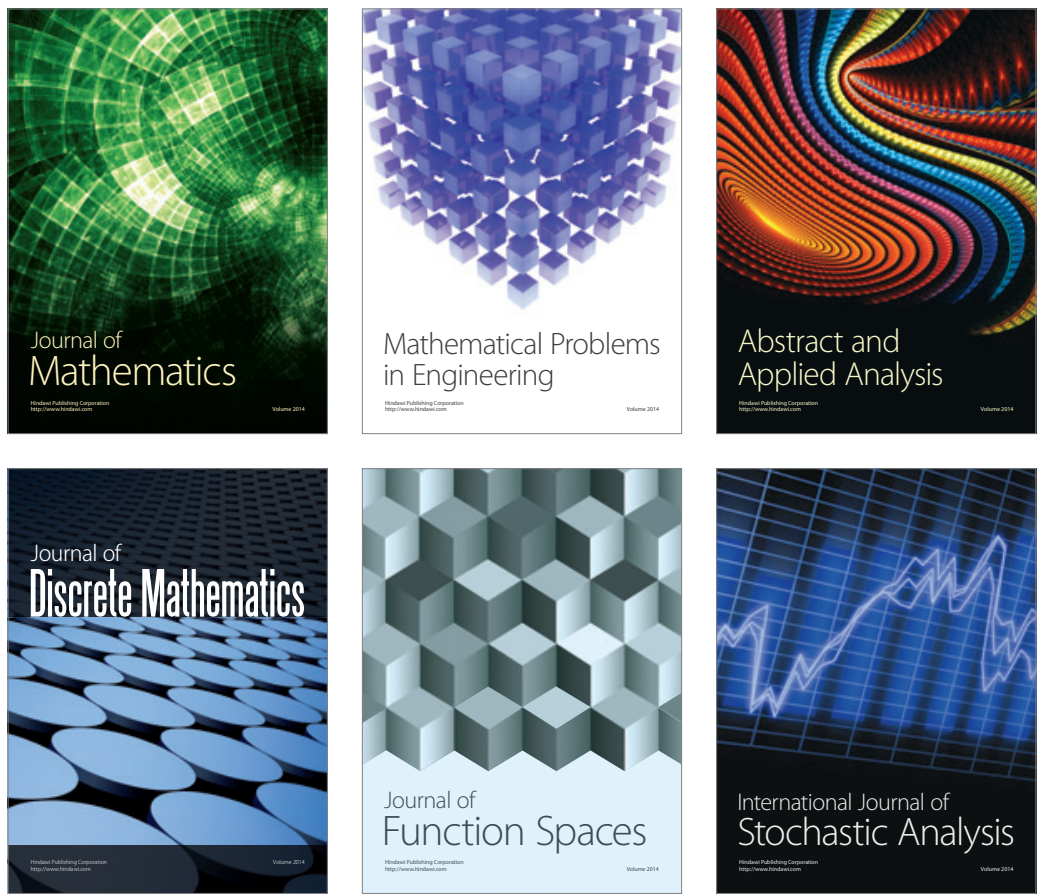

Journal of

Function Spaces

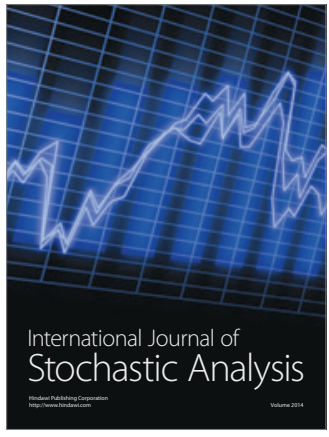

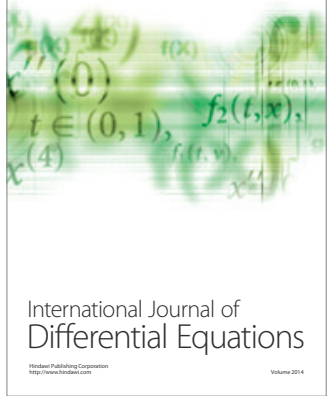
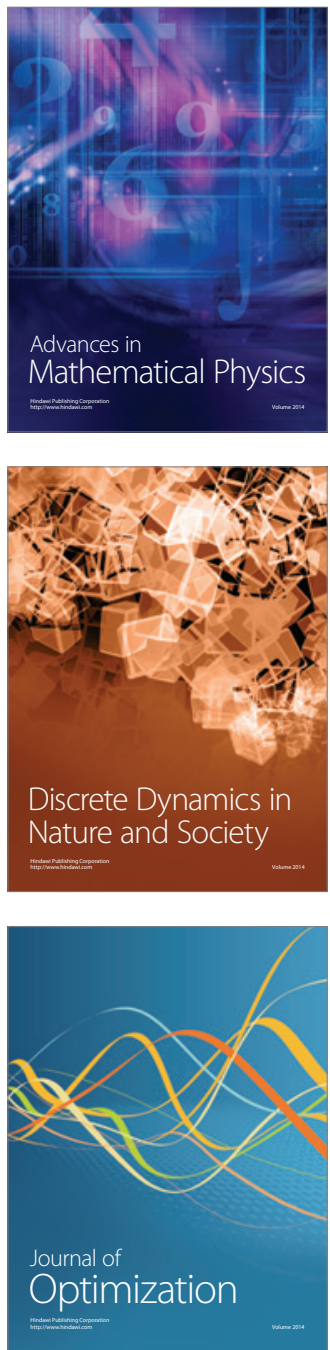